\title{
A Novel Shared Protection Scheme Based on Aggregate Wavelength in High Speed Networks
}

\author{
Anshu Oberoi, ${ }^{1}$ J. S. Sohal, ${ }^{2}$ and R. S. Kaler ${ }^{3}$ \\ ${ }^{1}$ Kanya Maha Vidyalaya, Jalandhar 144008, India \\ ${ }^{2}$ Ludhiana College of Engineering and Technology, Ludhiana, Punjab 141113, India \\ ${ }^{3}$ Thapar University, Patiala, Punjab 147004, India \\ Correspondence should be addressed to Anshu Oberoi; anshukmv@gmail.com
}

Received 3 June 2014; Revised 19 October 2014; Accepted 29 October 2014; Published 20 November 2014

Academic Editor: Stefan Wabnitz

Copyright (C) 2014 Anshu Oberoi et al. This is an open access article distributed under the Creative Commons Attribution License, which permits unrestricted use, distribution, and reproduction in any medium, provided the original work is properly cited.

\begin{abstract}
We propose novel analytical model of dynamic link cost evaluation in IP over WDM networks. We suggest disjoint path algorithm for the primary and backup path based on wavelength aggregate information, to provide shared backup. We show the optimality of pair selected because of joint optimization of the pair paths. The shareable capacity factor is introduced to establish the effect of load balancing on resources. We compared our simulation results with that of separate protection at connection and showed improvement on resource utilization performance of the network. We also study the blocking probability of proposed scheme.
\end{abstract}

\section{Introduction}

Most of the present day research has been focusing on the quality of service (QoS) routing with survivable paths in IP over wavelength division multiplexing (WDM) networks. The transmission rate of a wavelength channel may be high over an optical carrier (OC) from OC192 to OC768 but the bandwidth requirement of the signal may vary from OC 1 to even lower. Also the sensitivity of the type of traffic may vary from real time applications to offline messages. Using the capabilities of GMPLS, proposals of restoration mechanisms are discussed in [1, 2]. Wason and Kaler [3] have suggested rerouting technique for dynamic restoration to improve on blocking. These techniques suggest mechanisms in which reserved paths called backup paths can be used to quickly transfer the data in case of any failure detection.

This gives rise to another challenge of provisioning two paths (disjoint in nature) to establish a request for bandwidth guaranteed LSP. Along with this sufficient resource management is to be ensured with minimum path setup and signaling delays [4]. Fast recovery time and reduced resource utilization are the two main criteria for determining the quality of protection based survivability mechanism. It suggested that link-based protection and path-based protection provide, respectively, a short recovery time and reduced use of resources. In [5] virtual-link mapping scheme and link-cost formulas are presented to encourage the appropriate routing selection and load balancing, which can also contribute to better resource utilization ratio and blocking ratio. The paper [6] studies an algorithm to localize any shared risk link group failure via monitoring-trails (m-trails) together with the working lightpaths. The problem of selecting an optimal set of working lightpaths is shown to be NP-complete and has limitations for dynamic traffic. A shared-path protection algorithm with correlated risk (SPP_CR) is proposed in [7], in flexible bandwidth optical networks. Based on the correlated risk, the routing can be diverse and the sharing in backup spectral resource will be restricted. Collection of network state information like path links, bandwidth, and risk protection by backup LSPs is stored in each node [8]. It also suggests information distribution for update and path determination with desired protection and bandwidth constraints are the prime requirements.

In [9], multicast traffic grooming problem is addressed using a heuristic approach to maximize the bandwidth utilization for a set of sessions in an optical WDM mesh topology. To increase the network resource utilization the resource 
sharing [10] should be considered when backup LSP's are computed.

For the best results on sharing the routing algorithms must have intelligent information [11]. Kodialam and Lakshman [12] have shown that even though the aggregate information case only provides aggregate link usage information, it is still possible to make exact backup bandwidth reservation at each link. Kini et al. [13] proposed heuristics where only one aggregate value per link is advertise with slight modification to IGP-TE protocols but does not exploit efficiently the bandwidth sharing. Saidi et al. [11] suggested point of local repair based algorithm which offered a high bandwidth sharing with aggregate information but used fixed separate pools of bandwidth for primary and backup on each link. Optimization of primary and backup LSPs collectively is needed to total resource utilization metrics whereas mostly they are dealt with independently till now.

In our proposed work algorithm for optimal disjoint path is proposed on a novel cost model. The shareable capacity factor is used to establish the effect of sharing on resources. Joint optimization of the primary and backup LSPs computation, in our work, showed improvement on resource utilization of the network, which has been treated independently till now to the best of our knowledge. Our simulations show lower blocking for higher traffic rates as well. The paper is organized as follows. Section 2 presents the heuristics of proposed algorithm (WASP) and the disjoint path cost model; Section 3 describes the simulation results and analysis; conclusion of the work is hence followed.

\section{WASP Algorithm}

2.1. Proposed Scheme. In this section we present the mechanism of Wavelength Aggregate Shareable Protection (WASP), where nodes maintain the relevant information locally and links make reservation, based on the aggregate information, on the arrival of any request. The explicit route for the primary is computed at the ingress node and the chosen path is distributed for the setup of explicit disjoint backup path. The actual LSPs setup is done using signaling for the choice of optimal combination. We are considering backup paths to use sharing for the primary paths; this allows reserving a bandwidth " $b$," sufficient to meet the requirement of the maximum bandwidth LSP among the disjoint primary paths. Here we are considering only single link failure.

We consider the request for LSP to be defined as $\langle s, d$, $b, \lambda\rangle$ where $s, d$ refers to the source and destination node, respectively, $b$ is the bandwidth, and $\lambda$ is the wavelength. Our heuristics suggests a novel format for link capacity control table where any node " $a$ " with " $n$ " number of outgoing links maintains " $n$ " number of link control tables. The number of rows in each table corresponds to the number of wavelength channels in each link, which is assumed to be constant in our network. Any entry in this table tuple has fields 〈wavelength, conn_id, $\left.\max \left(\mathrm{BW}_{\mathrm{BP}}\right), R_{\mathrm{C}}\right\rangle$ where, against the request at a given wavelength, "conn_id" contains the list of connection identities of the primary paths for which the link reserves the backup, "max $\left(\mathrm{BW}_{\mathrm{BP}}\right)$ " refers to the maximum bandwidth reserved for the backup on that wavelength link, and " $R_{C}$ " gives the residual bandwidth on it. It is important to note that no record of the number of primary LSPs or the bandwidth occupied by them is needed to be maintained by the said table.

Considering there is a connection request between nodes " $s$ " and " $d$ " at any time " $t$ ", the primary is selected as per the shortest cost route. It is suggested to select a backup for which the source node distributes the control message of the minimum cost primary lsp found and the "conn_id" identifies the disjoint path required. "max" $\left(\mathrm{BW}_{\mathrm{BP}}\right)$ fix up the cost of the link through the node at the requested wavelength using the cost function proposed in Section 2.1 of this paper. The residual capacity of the link fixes the dynamic shareable potential of the link depending upon the value of " $\varepsilon$," the shareable capacity factor for the domain.

The new entries are made in the link control table for requested wavelength for optimal pair of primary and backup $\left\{\mathbf{P}^{\prime}, \mathbf{S}^{\prime}\right\}$ through signaling and the resources are reserved as

$$
R c=R_{C}-b_{i}
$$

[for primary lsp nodes]

if $\left(\max \left(\mathrm{BW}_{\mathrm{BP}}\right)<b_{i}\right)$ then

$$
\begin{gathered}
\max \left(\mathrm{BW}_{\mathrm{BP}}\right)=b_{i} \\
R c=R_{\mathrm{C}}-b_{i}
\end{gathered}
$$

[for backup lsp nodes],

where " $b_{i}$ " is the bandwidth required for " $i$ th" request.

On release of a connection, connection identity is sent along the backup. This control message updates the connection list of the table and residual capacity. The maximum backup reserved cell is changed only if the request had the maximum bandwidth among the shared protected primaries.

2.2. Disjoint Path Cost Model and Algorithm. The amount of the sharing possible on the backup path influences the choice of the primary path; hence the primary cannot be independently chosen at the outset. Then before routing computation of $(\mathbf{P}, \mathbf{S})$, the cost for each link $l \in L$ in the domain is set. Consider the fiber link has " $N$ " wavelength channels given as $\lambda_{t}$ of equal capacity $C, \forall 1 \leq t \leq N$. For any request " $i$," having bandwidth demand of $b_{i}$, at $\lambda_{I}$, the cost $a_{u v}$ to use a link $(u, v)$ on the primary path $\mathbf{P}$ is suggested as

$$
a_{u v}= \begin{cases}b_{i} & \text { if } R_{C}-b_{i} \geq 0 \text { at } \lambda_{I} \\ k b_{i} & \text { if } R_{C}-b_{i} \geq 0 \text { at } \lambda_{\neq I} \\ \infty & \text { otherwise }\end{cases}
$$

where $k>1$.

It can be seen that if the reservation is allowed by the residual bandwidth at the requested wavelength (following wavelength continuity), the cost incurred would be minimal. Else the cost involves a predefined increment because of 
the wavelength conversion to some of the other allowed channel of the link. The cost of the link becomes very high (suggested as $\infty$ in (3)) if it cannot accommodate the request on any of its channels. This model provides dynamic cost to each link in proportion to the demand raised through the request. It will provide a boon in selecting optimal path during disjoint pair selection algorithm, suggested in the later section of this paper.

The cost $c_{a b}$ to use a link $(a, b)$ on the backup path $\mathbf{S}$ is as

$$
c_{a b}= \begin{cases}0 & \text { if }(a, b) \notin \mathbf{P}_{i} \cap b_{i} \leq \max \left(\mathrm{BW}_{\mathrm{BP}}\right)_{a b} \\ b_{i} & \text { if }(a, b) \notin \mathbf{P}_{i} \cap \max \left(\mathrm{BW}_{\mathrm{BP}}\right)_{a b}<b_{i} \leq \varepsilon * C_{I} \\ k b_{i} & \text { if }(a, b) \notin \mathbf{P}_{i} \cap \max \left(\mathrm{BW}_{\mathrm{BP}}\right)_{a b}<b_{i} \leq \varepsilon * C_{\neq I} \\ \infty & \text { otherwise, }\end{cases}
$$

where $k>1$.

The equation above suggests that any link $(a, b)$ which is disjoint with the primary and can accommodate the reservation for backup within the already reserved bandwidth has the highest priority for selection and hence has zero cost. For otherwise disjoint link the cost of reservation includes either the higher bandwidth demand at wavelength continuity or wavelength conversion cost through factor " $k$." The link is rejected if not disjoint with the primary.

Algorithm 1. Given a directed graph $\mathbf{G}(\mathbf{E}, \mathbf{V})$ and a source destination pair $(s, d)$.

Step 1. Find shortest path $\mathbf{P}$ obeying the weight factor given in (3). If $\mathbf{P}$ doesn't exist then stop.

Step 2. Reserve the direction of all the links on the shortest path $\mathbf{P}$ and set the weights according to (4). A modified graph $\mathbf{G}^{\prime}$ is created.

Step 3. Find shortest path $\mathbf{S}$ in modified graph. If $\mathbf{S}$ doesn't exist, then stop.

Step 4. Make the union of $\mathbf{P}$ and $\mathbf{S}$, remove from the union the $\mathbf{P}$ links whose reversed links appear on $\mathbf{S}$ and vice-versa. Then regroup the left links into a set of two $\left\{\mathbf{P}^{\prime}, \mathbf{S}^{\prime}\right\}$ such that

$$
\mathbf{P}^{\prime} \cup \mathbf{S}^{\prime}=(\mathbf{P} \cup \mathbf{S})-\{\mathbf{P} \widetilde{\cap} \mathbf{S}\} .
$$

Step 5. $\left\{\mathbf{P}^{\prime}, \mathbf{S}^{\prime}\right\}$ give the required result.

The optimality of the solution hence obtained is derived using minicost flow theory. The choice of zero cost for reversed $\mathbf{P}$ links in $\mathbf{G}^{\prime}$ rather than negative values ensures loop free path selection. The novel cost model suggested through (3) and (4) introduces the distributed mechanism for path selection where the control tables at each node assign weight to links dynamically.

\section{Results and Discussion}

3.1. Simulation Setup. We simulate a dynamic environment over our example NSFnet network (Figure 1) with 14 nodes

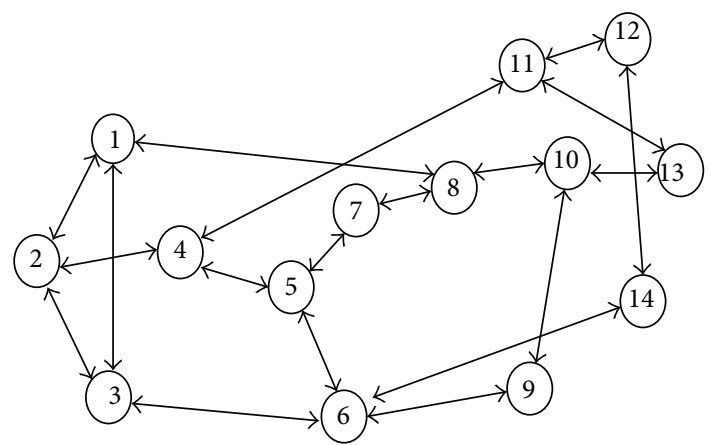

FIGURE 1: NSFNet network topology (14 nodes 19 links).

and 19 bidirectional links. We assume 16 wavelengths per link and the capacity of each wavelength " $C_{I}$ " is 300 units. The connection arrival process is Poisson and the connection holding time follows a negative Exponential distribution. The number of the connection requests follows the uniform distribution ranging from the full bandwidth request to unit value. Connection request is uniformly distributed among all the wavelength conversion ability. The average connection holding time is normalized to unity and the initial cost of each link is also assumed to be unity.

To evaluate the performance of our route-computation heuristics (WASP) we used resource utilization ratio metric " $\rho(t, \lambda)$," which is defined by the amount of allocated resources in terms of wavelength channels.

3.2. Results and Analysis. We define here " $\varepsilon$ " as the ratio of the total backup capacity reserved to that of the total primary capacity reserved on each link. We performed discrete event simulations for $\varepsilon$ in the range $(0,1)$ and investigated the effect on the resource utilization. We established the improvement of sharing the backup path by varying the value of " $\varepsilon$ "; Figure 3 shows the variation of resource utilization by the network at three different values. The decision on this value allows flexibility in making the choice on bandwidth share of the backup over a link. This can further be used to achieve control over the resource utilization with respect to the dynamic traffic demand. The lower ratio, $\varepsilon=0.2$, in our case, performs fairly well but not better when $\varepsilon=0.5$. This is because lesser reserved capacity would lead to the search of alternate paths which could be longer and hence not optimal for resource conservation, whereas the lower performance with high $\varepsilon, 0.8$ in our case, the routing process would rather become independent of sharing and longer primary paths as well.

We obtained the value of $\rho(t, \lambda)$ as against network load (in Erlangs) which is connection arrival rate multiplied with average holding time times a connection's bandwidth normalized in the unit of OC-192. We introduced $W_{\lambda}$ as the weighted balancing factor. We define it as the ratio of the aggregate residual capacity of the link to that of the total capacity. It is important to note here that the wavelength wise residual is maintained in the control table. We have used $k=2$, in (3) and (4), to include the cost effect of wavelength conversion, 


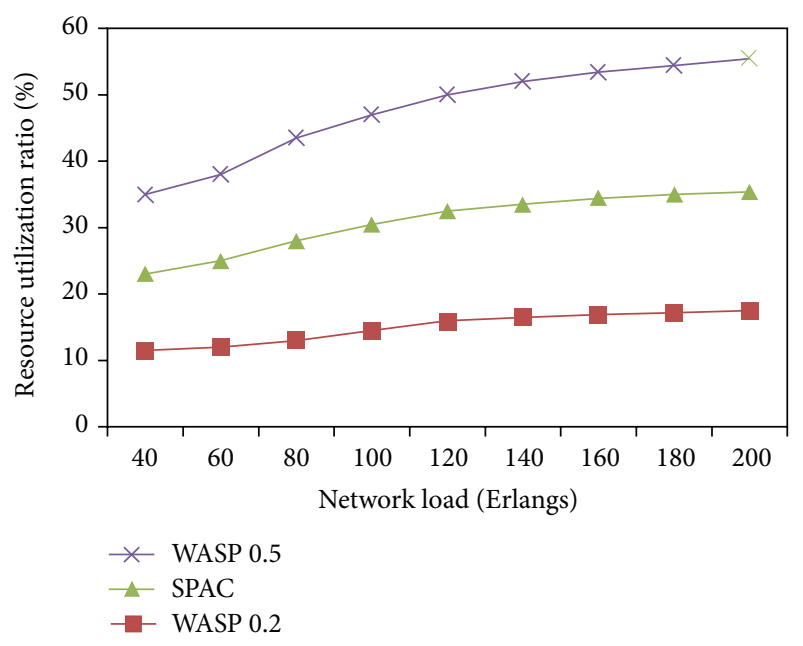

FIGURE 2: Resource utilization ratio versus network load.

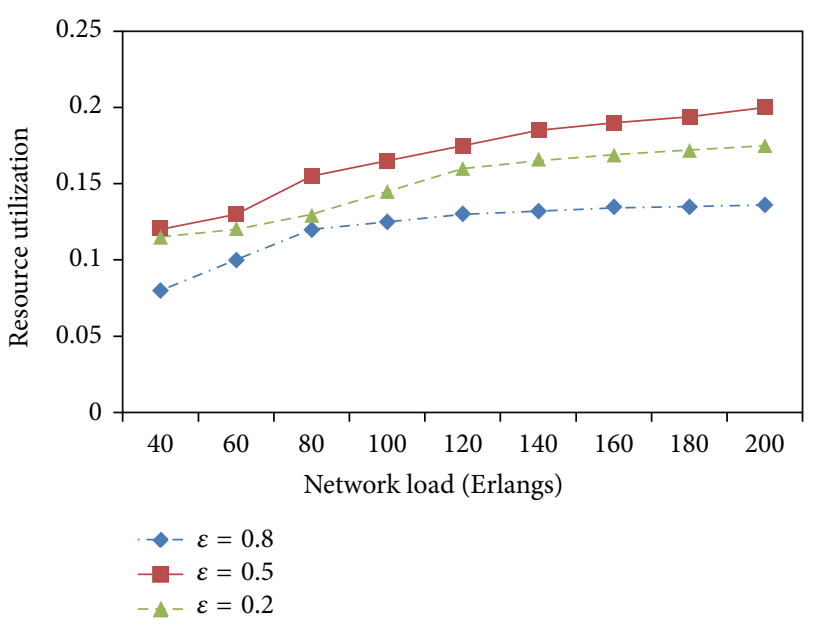

FIGURE 3: Resource utilization for varied $\varepsilon$.

if required, for link selection in any of the primary or backup paths.

$\rho(t, \lambda)$ measures how efficiently resources have been used. We compared our results, shown in Figure 2, with the separate protection at connection (SPAC) [14], against varied network load. Our heuristics performs better from the fact that having dynamic link weight generated through novel optimal cost model spreads out the load. The backup path choice is based on the network state and is not independent of the primary as given in the cost model. The model includes the connection burden on the present state of the network for cost fixation in distributed manner. Also the choice of the primary and backup as a path pair for optimality rather than the individual path optimization leads to better resource utilization as compared to [12] where the cost optimization of individual primary and backup is suggested. Further because of the aggregate information used to evaluate the cost of candidate links our proposed scheme has lesser complexity and smaller control update even at the release of the connection. The fixation of the bandwidth allocation for

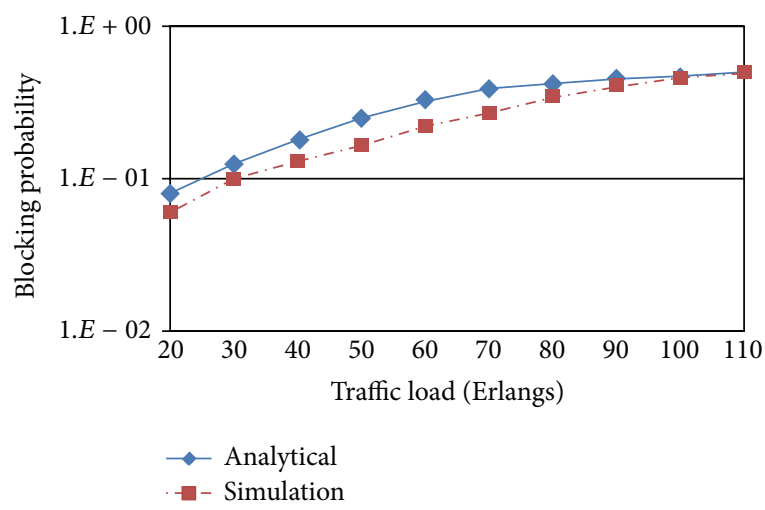

FIGURE 4: Blocking probability with network traffic load.

the primary and backup in the heuristics given in [11] does not allow flexibility in traffic grooming whereas in the heuristics presented in this paper factor $\varepsilon$ has to control the depth of sharing dynamically.

To further study the performance of our proposed scheme we study the blocking probability with varying load. Birman [15], blocking probability formula for a network having "l" links, all having $C$ channels (wavelengths) with Poisson arrivals while holding times are exponentially distributed with the unit mean, is used to analytically find the blocking probability using Erlangs B formula for the loss function. For the simulated results, the ratio of the blocked requests to the total requests generated over a specific time of simulation is used to obtain the blocking probability. The results are the average of 10 simulations done over 1000 virtual seconds of the simulation time. The analytical and simulation results are compared as shown in Figure 4; we achieved appreciable low blocking for low traffic. Though this is not the main aim of the proposed scheme to achieve target blocking as low as 103 , the scheme performs fairly well. Even at higher traffic the blocking is well below 1 , which suggests the efficiency of path selection of the proposed algorithm with judicious resource reservation. Still higher load is not shown in the graph as the comparison pattern became very similar.

\section{Conclusion}

We investigated the shared survivability problem for WDM mesh network in a dynamic context for single link failure based on a novel cost model. We suggested (WASP) the aggregate path state information for the nodes on a wavelength basis. This leads to dynamic routing of primary and backup path reservation in an optimal manner as the cost of both paths is decided on the basis of connection demand from the network. Our scheme suggests that the choice of backup path cannot be made independent of that of the primary for higher resource efficiency. Our proposed scheme is simpler in implementation because of few control messages required for modifications to the link control table. Simulation results show the higher resource utilization ratio as compared to separate path optimization. In this paper we considered the protection for every request though it can be based on class of 
service for further improvement in resource utilization. Also targeting still lower blocking is suggested as our future work.

\section{Conflict of Interests}

The authors declare that there is no conflict of interests regarding to the publication of this paper.

\section{References}

[1] R. K. Ahuja, T. L. Magnanti, and J. B. Orlin, Network Flows: Theory, Algorithms and Applications, Prentice Hall, Englewood Cliffs, NJ, USA, 1993.

[2] V. Sharma and F. Hellstrand, "Framework for MPLS based recovery," RFC 3469, February 2003.

[3] A. Wason and R. S. Kaler, "Rerouting technique with dynamic traffic in WDM optical networks," Optical Fiber Technology, vol. 16, no. 1, pp. 50-54, 2010.

[4] H. Drid, N. Ghani, and B. Cousin, "Application-aware protection in DWDM optical networks," Photonic Network Communications, vol. 22, no. 3, pp. 240-244, 2011.

[5] L. Tang, M. Cai, B. Li, and R. Wu, "A novel multi-link faulttolerant algorithm for survivability in multi-domain optical networks," Photonic Network Communications, vol. 24, no. 2, pp. 77-85, 2012.

[6] W. He, P.-H. Ho, B. Wu, and J. Tapolcai, "On identifying SRLG failures in all-optical networks," Optical Switching and Networking, vol. 10, no. 1, pp. 77-88, 2013.

[7] J. Zhang, C. Lv, Y. Zhao et al., "A novel shared-path protection algorithm with correlated risk against multiple failures in flexible bandwidth optical networks," Optical Fiber Technology, vol. 18, no. 6, pp. 532-540, 2012.

[8] P. Pan, G. Swallow, and A. Atlas, "Fast reroute extensions to RSVP-TE for LSP tunnels," RFC 4090, May 2005.

[9] A. K. Pradhan, S. Barat, and T. De, "A heuristic approach for multicast traffic grooming in optical WDM mesh networks," International Journal of Computer Network and Information Security, no. 1, pp. 39-47, 2014.

[10] L. Guo, J. Wu, W. Hou, and Y. Li, "Multicast protection algorithms based on aggregated logical topology in survivable multi-domain optical networks," Optik, vol. 123, no. 6, pp. 521526, 2012.

[11] M. Y. Saidi, B. Cousin, and J.-L. Le Roux, "PLR-based heuristic for backup path computation in MPLS networks," Computer Networks, vol. 53, no. 9, pp. 1467-1479, 2009.

[12] M. Kodialam and T. V. Lakshman, "Dynamic routing of restorable bandwidth-guaranteed tunnels using aggregated network resource usage information," IEEE/ACM Transactions on Networking, vol. 11, no. 3, pp. 399-410, 2003.

[13] S. Kini, K. Kodialam, and T. V. Lakshaman, "Shared backup label switch path restoration," Internet Draft IETF, 2001.

[14] C. Ou, K. Zhu, H. Zang, L. H. Sahasrabuddhe, and B. Mukherjee, "Traffic grooming for survivable WDM networks-shared protection," IEEE Journal on Selected Areas in Communications, vol. 21, no. 9, pp. 1367-1383, 2003.

[15] A. Birman, "Computing approximate blocking probabilities for a class of all-optical networks," IEEE Journal on Selected Areas in Communications, vol. 14, no. 5, pp. 852-857, 1996. 

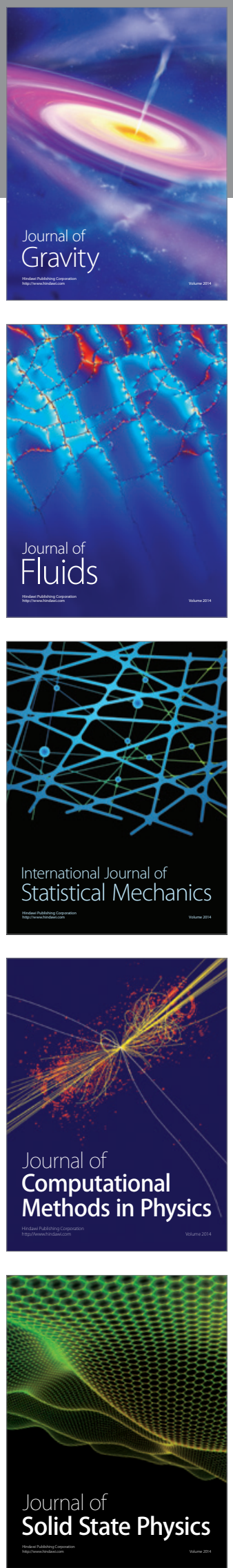

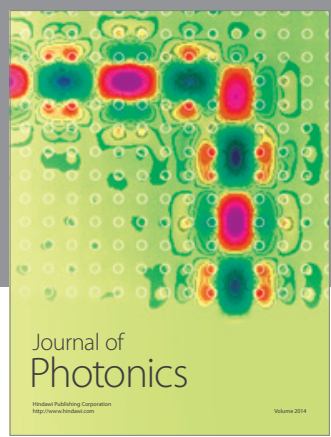

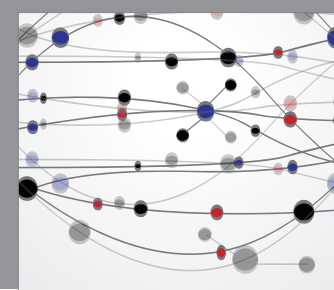

The Scientific World Journal

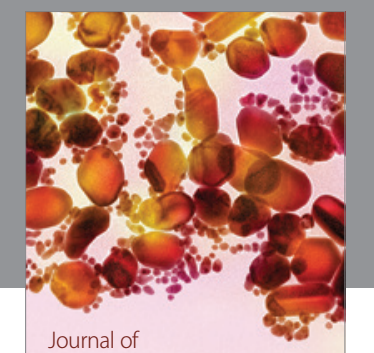

Soft Matter
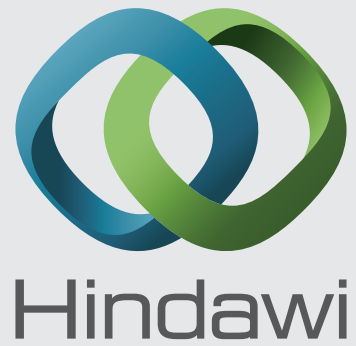

Submit your manuscripts at

http://www.hindawi.com
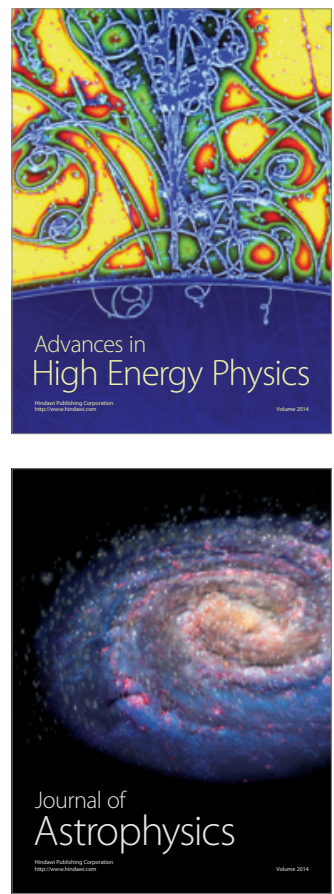
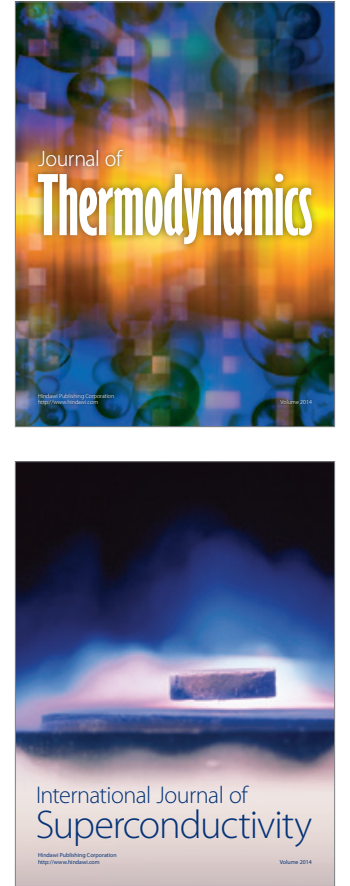
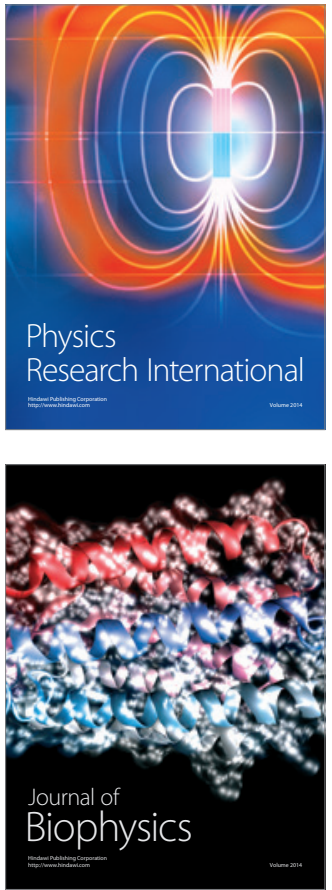
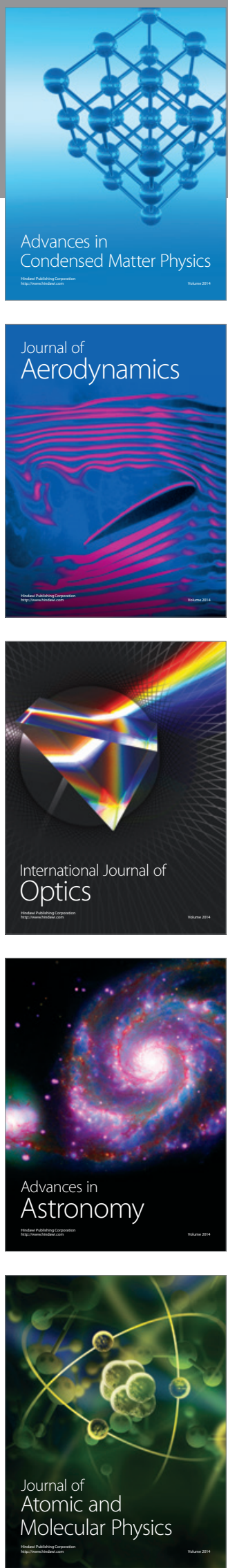